\title{
Ciencia de implementación: Una nueva estrategia para mejorar la práctica clínica en sectores de salud con escasos recursos
}

\author{
Implementation science: A new strategy to improve clinical practice in low-income health sectors \\ Doriam E. Camacho Rodriguez ${ }^{1,2}$, Karen A. Dominguez-Cancino ${ }^{2,3}$, Patrick A. Palmieri ${ }^{3,4,5}$, Juan M. Leyva- \\ Moral $^{3,6}$
}

\section{RESUMEN}

Objetivo: Proporcionar una visión general sobre el campo emergente de la ciencia de la implementación a los profesionales de salud en Latinoamérica. Materiales y Métodos: Revisión rápida de evidencias para definir la ciencia de la implementación, describir su estado, presentar los marcos conceptuales más citados en la literatura, y proveer recomendaciones para la educación en ciencias de la salud. Resultados: La ciencia de la implementación es una nueva, pero ampliamente reconocida, ciencia con una definición formal y teorías que la apoyan. Los dos marcos conceptuales más ampliamente citados en la literatura son el Promoting Action on Research Implementation in Health Services (PARiHS) y el Consolidated Framework for Implementation Research (CFIR). Sin embargo, se observa que ambos marcos conceptuales requieren una mejora continua para abordar los vínculos entre investigación y práctica. Específicamente, a medida que la ciencia se desarrolla, es necesario prestar más atención a la vinculación de las ciencias de descubrimiento, traducción e implementación en un enfoque de ciencia aplicada para transformar el conocimiento en evidencia comprensible para su uso inmediato en la práctica diaria. Conclusiones: La ciencia de la implementación tiene el potencial para acelerar el rol de la mejora de la calidad en el sector público de salud. La evidencia puede ser rápidamente incorporada en la práctica clínica con la presencia de profesionales de salud preparados para este rol emergente. De igual forma, los programas de ciencias de la salud en las Universidades de Latinoamérica necesitan empezar a explorar la utilidad de desarrollar grados académicos doctorales de práctica para apoyar la mejora del sistema de salud a través de la ciencia de la implementación.

Palabras clave: ciencia de la implementación, práctica basada en la evidencia, prestación de atención de salud, mejoramiento de la calidad

\begin{abstract}
Objetivo: Proporcionar una visión general sobre el campo emergente de la ciencia de la implementación a los profesionales de salud en Latinoamérica. Materiales y Métodos: Revisión rápida de evidencias para definir la ciencia de la implementación, describir su estado, presentar los marcos conceptuales más citados en la literatura, y proveer recomendaciones para la educación en ciencias de la salud. Resultados: La ciencia de la implementación es una nueva, pero ampliamente reconocida, ciencia con una definición formal y teorías que la apoyan. Los dos marcos conceptuales más ampliamente citados en la literatura son el Promoting Action on Research Implementation in Health Services (PARiHS) y el Consolidated Framework for Implementation Research (CFIR). Sin embargo, se observa que ambos marcos conceptuales requieren una mejora continua para abordar los vínculos entre investigación y práctica. Específicamente, a medida que la ciencia se desarrolla, es necesario prestar más atención a la vinculación de las ciencias de descubrimiento, traducción e implementación en un enfoque de ciencia aplicada para transformar el conocimiento en evidencia comprensible para su uso inmediato en la práctica diaria. Conclusiones: La ciencia de la implementación tiene el potencial para acelerar el rol de la mejora de la calidad en el sector público de salud. La evidencia puede ser rápidamente incorporada en la práctica clínica con la presencia de profesionales de salud preparados para este rol emergente. De igual forma, los programas de ciencias de la salud en las Universidades de Latinoamérica necesitan empezar a explorar la utilidad de desarrollar grados académicos doctorales de práctica para apoyar la mejora del sistema de salud a través de la ciencia de la implementación.
\end{abstract}

Palabras clave: ciencia de la implementación, práctica basada en la evidencia, prestación de atención de salud, mejoramiento de la calidad 
${ }^{1}$ Universidad Cooperativa de Colombia. Escuela de Enfermería. Colombia.

${ }^{2}$ EnfAmericas Research Center. Asociación Peruana de Enfermería. Lima - Perú

${ }^{3}$ Center for Health Sciences Research. Universidad María Auxiliadora. Lima - Perú

${ }^{4}$ Universidad Norbert Wiener. Escuela de Enfermería. Lima - Perú

${ }^{5}$ College of Graduate Health Studies. A.T. Still University. MO. USA

${ }^{6}$ Universitat Autonoma de Barcelona. Faculty of Medicine. Department of Nursing. Spain

\section{INTRODUCCIÓN}

A pesar de que se han generado miles de evidencias críticas, relevantes para mejorar la práctica clínica a través de la ciencia del "descubrimiento", muchas de ellas han sido transformadas recientemente en evidencias comprensibles para el consumo clínico a través de la ciencia de la "traducción". Sin embargo, la evidencia traducida habitualmente no se aplica para mejorar la práctica clínica, por medio de guías y protocolos. La brecha entre la evolución del conocimiento con sus hallazgos y la transformación de la evidencia para su implementación en nuevas prácticas clínicas ha resultado en un cuidado inapropiado en los diferentes países e instituciones (1-3). Aunque la traducción de la ciencia mueve los hallazgos de la investigación a evidencia utilizable, no existe claridad sobre cómo implementar la evidencia en la práctica clínica diaria. Implementar nuevo conocimiento en los complejos sistemas adaptativos existentes (4) requiere de profesionales clínicos con nuevas habilidades y destrezas en la gestión de proyectos (5), incluyendo planificación, liderazgo, evaluación y reflexión.

La investigación no sólo debe ser publicada en revistas, sino también ser transformada de forma eficiente y efectiva en evidencia útil para su implementación rápida en la práctica clínica diaria. A pesar de los billones de dólares invertidos en investigación en el mundo (6), sólo la mitad de la evidencia alguna vez alcanza una difusión generalizada en la práctica clínica (7). Con intercambios inadecuados de conocimiento entre investigadores, clínicos, y normas profesionales $(8,9)$, la recepción de la evidencia requiere una media de 17 años para ser integrada en la práctica clínica $(10,11)$. Como resultado, la ciencia de la implementación está emergiendo con soluciones contemporáneas para llevar la evidencia a la práctica clínica $(12,13)$.

La ciencia de la implementación o investigación para la implementación puede ser definida como "el estudio de los métodos para promover la aceptación de los hallazgos de la investigación y otras prácticas basadas en evidencia como prácticas rutinarias, y, por lo tanto, mejorar la efectividad de los servicios de salud.." (14). Dicho objetivo de mejorar la salud de las personas, se logra a través de la aplicación equitativa y eficaz de un conocimiento científico rigurosamente evaluado, que implica la identificación de las evidencias que hayan producido un impacto positivo en la salud de la comunidad (15). Dicha ciencia está generando marcos referenciales, modelos y estrategias para unir la diseminación de la investigación con la implementación de los hallazgos (16) y para ello hace un estudio sistemático de las intervenciones, servicios o programas que han demostrado ser efectivos en diversos contextos (17).

Los nuevos profesionales de la salud en práctica educados con un énfasis en la ciencia de la implementación son competentes para traducir los hallazgos de la investigación e implementar habilidades de práctica basada en evidencia en las profesiones. De acuerdo al Instituto de Salud de Estados Unidos (18), "Todos los profesionales de salud deberían ser educados en la entrega de cuidado centrado en el paciente como miembros de un equipo interdisciplinario, enfatizando en la práctica basada en evidencia, con enfoque en la mejora de la calidad, e informática" (p. 45). Como tal, los científicos de la implementación podrían estar abordando el problema con grandes inversiones públicas en investigación que fallan en producir los beneficios sociales esperados (19).

Con la ayuda de la ciencia para la implementación se procura aplicar una investigación sistemática que permita la medición y evaluación de resultados obtenidos a partir de análisis científicos rigurosos, para identificar los desafíos en la replicación y adaptación a gran escala y en distintos entornos, de intervenciones basadas en evidencia (20). La literatura refiere la necesidad de que en dicha implementación se considere la importancia de los usuarios, los prestadores de servicios de salud, las organizaciones y los responsables de la formulación de políticas en salud (21).

\section{TEORÍAS DE IMPLEMENTACIÓN}

La ciencia de la implementación ofrece a los clínicos teorías robustas (22) para guiar el intercambio de conocimiento, y los procesos interactivos y sistémicos, responsables de 
estimular el cambio y la innovación en las organizaciones (23). El conocimiento que se referencia en este intercambio puede originarse desde la teoría, investigación, práctica, y/o la experiencia (24). En el contexto de proyectos de mejora de la calidad (25), el conocimiento intercambiado usualmente se planifica a propósito, y la evidencia traducida es implementada en cambios específicos en la práctica clínica $(26,27)$. Dichos cambios son exitosos cuando se integran en la memoria de la organización a través de políticas, procedimientos, procesos y/o prácticas (28).

En el contexto de la ciencia de la implementación, el término "teoría" es usado para referirse a los marcos referenciales, modelos y estrategias que facilitan la implementación de los hallazgos de la investigación en la práctica clínica (29). En tal sentido, las teorías de implementación difieren de los modelos de práctica clínica, como el Modelo de Cuidado Crónico (30), y los otros modelos ya que abordan la brecha del saber hacer (31) con estrategias, métodos, y/o procesos para cambiar exitosamente la práctica clínica más que explicar los procesos de aplicación de cuidado y/o estrategias de evaluación dentro del contexto del sistema, organizaciones, y/o unidades (32); sin embargo, estos modelos habitualmente son incorporados en las teorías de implementación.

En general, las teorías de implementación se enfocan en la difusión de los hallazgos de investigación en la práctica clínica utilizando una variedad de estrategias de implementación(29) relacionadas con la traducción del conocimiento y el intercambio, la difusión de la innovación y la gestión del cambio, la teoría organizacional y de desarrollo, y los cuidados de salud basados en evidencia.(33) Estas teorías no sólo reconocen, sino también abordan las barreras atribuidas a las dificultades en la implementación que surgen en múltiples niveles del sistema de salud(34), incluyendo la persona atendida, los proveedores, la organización, el financiador, y las políticas (35).

La ciencia de la implementación se fundamenta en tres bases: 1) el conjunto de marcos o modelos que orientan sobre la mejor estrategia de implementación acorde al contexto de la práctica, dentro de los cuales destacan los Modelos Iowa (36) y Ottawa (37); 2) el marco explicativo necesario para entender los resultados de la implementación, como el Modelo COM-B (Capability Opportunity Motivation Behaviour System) (38) y la Teoría de la Difusión de Innovaciones (39); y 3) el modelo o marco que evalúa la implementación, como el Modelo PRECEDE-PROCEDE (Predisposing,
Reinforcing and Enabling Constructs in Educational Diagnosis and EvaluationPolicy, Regulatory, and Organizational Constructs in Educational and Environmental Development) (40). También existen modelos propuestos que abarcan todas las etapas de un proceso de implementación, partiendo de la evaluación de barreras y facilitadores y la adaptación de intervenciones a contextos específicos, hasta la definición de monitoreos efectivos y permanentes, como el Modelo RE-AIM (Reach, Effectiveness, Adoption, Implementation, and Maintenance) (41) y el Dynamic Adaption Process (42).

La mejor forma de implementar la investigación es difícil de definir, debido a que los cambios en la práctica clínica requieren modificaciones de comportamiento por parte de los profesionales clínicos (43); sin embargo, existen dos marcos referenciales que pueden ayudar a guiar la implementación de la evidencia, incluyendo el PARiHS (Promoting Action on Research Implementation in Health Services) y CFIR (Consolidated Framework for Implementation Research).

\section{Marco Referencial PARiHS}

El marco referencial PARiHS (Promoviendo la Acción en la investigación para su implementación en los Servicios de Salud) es la guía más antigua para trasladar la investigación a la práctica clínica $(44,45)$, y es el marco de referencia más citado en la literatura de ciencia de la implementación (Helfrich et al., 2010). Fue desarrollado hace más de 20 años (44), y ha evolucionado a un modelo actual a través de tres fases: Fase 1) Desarrollo y análisis del Concepto (1998-2002); Fase 2) Estudios empíricos de casos (2001-2003) y, Fase 3) Desarrollo de una herramienta de diagnóstico/evaluación (2003- al presente) (46). De acuerdo con Kitson et al., (45), "hay un pequeño, pero creciente cuerpo de evidencia desde la investigación y la práctica que muestra que el marco referencial PARiHS tiene integridad conceptual, validez de aspecto $y$ conceptual' (p. 8).

El marco referencial PARiHS provee una forma de implementar la investigación en la práctica. Para que el conocimiento sea implementado exitosamente en acciones, se debe gestionar la interacción de tres elementos clave: 1) Evidencia o nivel y naturaleza de la evidencia(47); 2) Contexto o ambiente en el que la investigación va a ser aplicada(48); y 3) Facilitación, o el método por el cual el proceso es facilitado.(49) Se cree que cada uno de los tres elementos son igualmente importantes, dado que la evidencia de 
alta calidad habitualmente no es aceptada en los espacios de práctica, debido a que su introducción no se adaptó al entorno ni a las partes interesadas. Es importante destacar, que este marco referencial continúa siendo revisado, probado y desarrollado (50), con propuestas de revisión al Marco Referencial basadas en evidencia, generando una guía de implementación que incluya herramientas de referencia (51).

\section{Marco Referencial CFIR}

El marco referencial CFIR (marco consolidado para la investigación de implementación) es una taxonomía con terminología y definiciones que crean una base de conocimiento para la implementación de factores a través de múltiples escenarios y contextos (52). Desde su publicación en 2009, el CFIR ha sido citado en más de 300 publicaciones (53). Basado en una amplia síntesis ampliamente citada de factores de implementación $(54,55)$, se utilizó un proceso de muestreo de bola de nieve para identificar un amplio rango de teorías relacionadas a la diseminación, innovación, cambio organizacional, implementación, traducción del conocimiento y recepción de investigación. A través de un riguroso análisis, los constructos teóricos fueron evaluados, definidos $\mathrm{y}$ operacionalizados en una taxonomía de cinco dominios que incluyen; 1) características de la intervención, como fuerza y calidad de la evidencia; 2) ajuste al medio, como las necesidades de los pacientes y los recursos; 3) Medio interno, como cultura y compromiso de liderazgo; 4) características individuales: como conocimientos y habilidades; y 5) proceso, como plan, evaluación y reflexión.(56) De acuerdo con Damschroder et al. (33), el CFIR es un marco referencial meta-teórico pragmático que puede ser usado para complementar teorías de procesos y modelos de evaluación.

\section{CONCLUSION}

Este documento proporciona una visión general sobre cómo ha surgido la ciencia de la implementación para trasladar los hallazgos de la investigación desde la difusión a la implementación en la práctica clínica. Las ciencias estudian los éxitos y fracasos relacionados con la aceptación de la evidencia en la práctica clínica, a través de disciplinas y entornos institucionales. Mediante la aplicación de teorías de implementación, los entornos interactivos, la autogestión, los procesos dinámicos y los sistemas adaptativos se define la compleja organización adaptativa como un constructo multifacético. En este sentido, los programas de doctorado profesional están preparando a los clínicos para aplicar las teorías de implementación, como PARiHS y CFIR, para mejorar las prácticas clínicas. Aplicando el conocimiento de la ciencia de la implementación, los investigadores de ciencias aplicadas pueden reducir la brecha entre los resultados originales de la investigación que resultan en la traducción de evidencia para informar la práctica guiada por la teoría.

\section{AUTOR DE CORRESPONDENCIA}

\author{
Dr .Patrick A. Palmieri \\ Escuela de Enfermería \\ Universidad Norbert Wiener \\ Teléfono: 98760853 \\ E-mail: patrick.palmieri@globalnursing.org
}

\section{REFERENCIAS BIBLIOGRÁFICAS}

1. Hussey PS, Anderson GF, Osborn R, Feek C, McLaughlin V, Millar J, et al. How does the quality of care compare in five countries? Health Affairs. 2004;23(3):89-99. https://doi.org/10.1377/hlthaff.23.3.89

2. McGlynn EA, Asch SM, Adams J, Keesey J, Hicks J, DeCristofaro A, et al. The quality of health care delivered to adults in the United States. NEJM. 2003;348(26):2635-45. https://doi.org/10.1056/NEJMsa022615

3. Seddon ME, Marshall MN, Campbell SM, Roland MO. Systematic review of studies of quality of clinical care in general practice in the UK, Australia and New Zealand. Quality in Health Care. 2001;10(3):152-8. https://doi.org/10.1136/qhc.0100152..

4. Barach P, Johnson JK. Understanding the complexity of redesigning care around the clinical microsystem. Quality and Safety in Health Care. 2006;15(suppl 1):i10-i6. https://doi.org/10.1136/qshc.2005.015859

5. Kuziemsky C. Decision-making in healthcare as a complex adaptive system. Healthcare Management Forum. 2016;29(1):4-7. https://doi.org/10.1177/0840470415614842

6. Moses H, Matheson DHM, Cairns-Smith S, George BP, Palisch C, Dorsey ER. The anatomy of medical research: US and international comparisons. JAMA. 
2015;313(2):174-89.

https://doi.org/10.1001/jama.2014.15939

7. Balas EA, Boren SA. Managing clinical knowledge for health care improvement. Yearb Med Inform. 2000;09(01):65-70. https://doi.org/10.1055/s-0038-1637943

8. Grimshaw JM, Eccles MP, Lavis JN, Hill SJ, Squires JE. Knowledge translation of research findings. Implementation Science. 2012;7(1):50. https://doi.org/10.1186/17485908-7-50

9. Smith KE. The politics of ideas: The complex interplay of health inequalities research and policy. Science and Public Policy. 2013;41(5):561-74. https://doi.org/10.1093/scipol/sct085

10. Grant J, Green L, Mason B. Basic research and health: a reassessment of the scientific basis for the support of biomedical science. Research Evaluation. 2003;12(3):217-24. https://doi.org/10.3152/1471544037817766 18

11. Morris ZS, Wooding S, Grant J. The answer is 17 years, what is the question: Understanding time lags in translational research. Journal of the Royal Society of Medicine. 2011;104(12):510-20. https://doi.org/10.1258/jrsm.2011.110180

12. Rapport F, Clay-Williams R, Churruca K, Shih P, Hogden A, Braithwaite J. The struggle of translating science into action: Foundational concepts of implementation science. Journal of Evaluation in Clinical Practice. 2018;24(1):117-26. https://doi.org/10.1111/jep.12741

13. Süárez-Obando $\mathrm{F}$, Gómez-Restrepo $\mathrm{C}$, Castro-Díaz SM. Ciencias de la implementación: De la evidencia a la práctica. Acta Medica Colombiana. 2018;43(4):207-16. [Available from: http://www.redalyc.org/articulo.oa?id=1631 59058006].

14. Eccles MP, Mittman BS. Welcome to implementation science. Implementation Science. 2006;1(1):1. https://doi.org/10.1186/1748-5908-1-1

15. Rumbo JM, Martínez ÁA, Sobrido M, Raña $\mathrm{CD}$, Vázquez MV, Braña B. Implementar evidencias e investigar en implementación: Dos realidades diferentes y prioritarias. Enfermería Clinica. 2016;26(6):381-6. https://doi.org/10.1016/j.enfcli.2016.10.002

16. Graham ID, Logan J, Harrison MB, Straus SE, Tetroe J, Caswell W, et al. Lost in knowledge translation: time for a map? J Contin Educ Health Prof. 2006;26. https://doi.org/10.1002/chp.47
17. Gilson L, editor. Health policy and systems research: A methodology reader. Geneva, Switzerland: WHO Press; 2012. [Available from:

https://apps.who.int/iris/handle/10665/4480 3]

18. Greiner AC, Knebel E, editors. Health professions education: A bridge to quality. Washington, DC National Academies Press; 2004. https://doi.org/10.17226/10681

19. Tetroe JM, Graham ID, Foy R, Robinson N, Eccles MP, Wensing M, et al. Health research funding agencies' support and promotion of knowledge translation: An international study. The Milbank Quarterly. 2008;86(1):125-55.

https://doi.org/10.1111/j.1468-

0009.2007.00515.x

20. Zepeda KGM, Silva MMd, Silva ÍR, Redko C, Gimbel S. Fundamentos de la ciencia de la implementación: Un curso intensivo sobre un emergente campo de investigación. Escola Anna Nery Revista de Enfermagem. 2018;22(2):e20170323. https://doi.org/10.1590/2177-9465-ean2017-0323

21. McKillop A, Shaw J, Sheridan N, SteeleGray C, Carswell P, Wodchis WP, et al. Understanding the attributes of implementation frameworks to guide the implementation of a model of communitybased integrated health care for older adults with complex chronic conditions: A metanarrative review. International Journal of Integrated Care. 2017;17(2):10. https://doi.org/10.5334/ijic.2516

22. Lynch EA, Mudge A, Knowles S, Kitson AL, Hunter SC, Harvey G. "There is nothing so practical as a good theory": A pragmatic guide for selecting theoretical approaches for implementation projects. BMC Health Services Research. 2018;18(1):857. https://doi.org/10.1186/s12913-018-3671-z

23. van den Driessen Mareeuw F, Vaandrager L, Klerkx L, Naaldenberg J, Koelen M. Beyond bridging the know-do gap: a qualitative study of systemic interaction to foster knowledge exchange in the public health sector in The Netherlands. BMC Public Health. 2015;15(1):922.

https://doi.org/10.1186/s12889-015-2271-7

24. Green LW. Making research relevant: If it is an evidence-based practice, where's the practice-based evidence? Family Practice. 2008;25(suppl_1):i20-i4. https://doi.org/10.1093/fampra/cmn055 
25. Dixon-Woods M. Harveian Oration 2018: Improving quality and safety in healthcare Clinical Medicine. 2019;19(1):47-56. https://doi.org/10.7861/clinmedicine.19-147

26. Lomas J. The in-between world of knowledge brokering. BMJ. 2007;334(7585):129-32. https://doi.org/10.1136/bmj.39038.593380. $\mathrm{AE}$

27. Ward VL, House AO, Hamer S. Knowledge brokering: Exploring the process of transferring knowledge into action. BMC Health Services Research. 2009;9(1):12. https://doi.org/10.1186/1472-6963-9-12

28. Glasgow RE, Emmons KM. How can we increase translation of research into practice? Types of evidence needed. Annual Review of Public Health. 2007;28(1):413-33. https://doi.org/10.1146/annurev.publhealth. 28.021406.144145

29. Bauer MS, Damschroder L, Hagedorn H, Smith J, Kilbourne AM. An introduction to implementation science for the nonspecialist. BMC Psychology. 2015;3(1):32. https://doi.org/10.1186/s40359-015-0089-9

30. Wagner EH, Austin BT, Davis C, Hindmarsh M, Schaefer J, Bonomi A. Improving chronic illness care: Translating evidence into action. Health Affairs. 2001;20(6):64-78. https://doi.org/10.1377/hlthaff.20.6.64

31. Pakenham-Walsh N. Learning from one another to bridge the "know-do gap". BMJ. 2004;329(7475):1189. https://doi.org/10.1136/bmj.329.7475.1189

32. Nilsen P. Making sense of implementation theories, models and frameworks. Implementation Science. 2015;10(1):53. https://doi.org/10.1186/s13012-015-0242-0

33. Damschroder LJ, Aron DC, Keith RE, Kirsh SR, Alexander JA, Lowery JC. Fostering implementation of health services research findings into practice: a consolidated framework for advancing implementation science. Implementation Science. 2009;4(1):50. https://doi.org/10.1186/17485908-4-50

34. Paina L, Peters DH. Understanding pathways for scaling up health services through the lens of complex adaptive systems. Health Policy and Planning. 2011;27(5):365-73. https://doi.org/10.1093/heapol/czr054

35. Ferlie EB, Shortell SM. Improving the quality of health care in the United Kingdom and the United States: A framework for change. The Milbank Quarterly.
2001;79(2):281-315.

https://doi.org/10.1111/1468-0009.00206

36. Titler MG, Kleiber C, Steelman VJ, Rakel BA, Budreau G, Everett LQ, et al. The Iowa Model of Evidence-Based Practice to Promote Quality Care. Critical Care Nursing Clinics of North America. 2001;13(4):497$509 . \quad$ https://doi.org/10.1016/S08995885(18)30017-0

37. Logan J, Graham ID. Toward a comprehensive interdisciplinary model of health care research use. Science Communication. 1998;20(2):227-46. https://doi.org/10.1177/1075547098020002 004

38. Michie S, van Stralen MM, West R. The behaviour change wheel: A new method for characterising and designing behaviour change interventions. Implementation Science. 2011;6(1):42. https://doi.org/10.1186/1748-5908-6-42

39. Rogers EM. Diffusion of innovations. 5 ed. New York, NY: The Free Press; 2003.

40. Green LW, Kreuter MW. Health promotion planning: An educational and ecological approach. 4 ed. New York, NY: McGrawHill; 2005.

41. Glasgow RE, Vogt TM, Boles SM. Evaluating the public health impact of health promotion interventions: The RE-AIM framework. American Journal of Public Health. $\quad$ 1999;89(9):1322-7. https://doi.org/10.2105/ajph.89.9.1322

42. Brownson R, Colditz G, Proctor E. Dissemination and implementation research in health. 2 ed. New York, NY: Oxford University Press,; 2018.

43. Khalil $H$. Knowledge translation and implementation science: what is the difference? International Journal of Evidence-Based Healthcare. 2016;14(2):3940.

https://doi.org/10.1097/xeb.0000000000000 086

44. Kitson A, Harvey G, McCormack B. Enabling the implementation of evidence based practice: A conceptual framework. Quality in Health Care. 1998;7(3):149-58. https://doi.org/10.1136/qshc.7.3.149

45. Kitson AL, Rycroft-Malone J, Harvey G, McCormack B, Seers K, Titchen A. Evaluating the successful implementation of evidence into practice using the PARiHS framework: theoretical and practical challenges. Implementation Science. 2008;3(1):1. https://doi.org/10.1186/17485908-3-1 
46. National Collaborating Centre for Methods and Tools. PARiHS framework for implementing research into practice 2019 [Available from: https://www.nccmt.ca/knowledgerepositories/search/85].

47. Rycroft-Malone J, Harvey G, Seers K, Kitson A, McCormack B, Titchen A. An exploration of the factors that influence the implementation of evidence into practice. Journal of Clinical Nursing. 2004;13(8):913$24 . \quad$ https://doi.org/10.1111/j.13652702.2004.01007.x

48. McCormack B, Kitson A, Harvey G, Rycroft-Malone J, Titchen A, Seers K. Getting evidence into practice: The meaning of context. Journal of Advanced Nursing. 2002;38(1):94-104.

https://doi.org/10.1046/j.1365-

2648.2002.02150.x

49. Harvey G, Loftus-Hills A, Rycroft-Malone J, Titchen A, Kitson A, McCormack B, et al. Getting evidence into practice: The role and function of facilitation. Journal of Advanced Nursing. https://doi.org/10.1046/j.13652648.2002.02126.x

50. Harvey G, Kitson A. PARIHS revisited: from heuristic to integrated framework for the successful implementation of knowledge into practice. Implementation Science. 2016;11(1):33.

https://doi.org/10.1186/s13012-016-0398-2

51. Stetler CB, Damschroder LJ, Helfrich CD, Hagedorn HJ. A Guide for applying a revised version of the PARIHS framework for implementation. Implementation Science. 2011;6(1):99. https://doi.org/10.1186/17485908-6-99

52. Keith RE, Crosson JC, O'Malley AS, Cromp D, Taylor EF. Using the Consolidated Framework for Implementation Research (CFIR) to produce actionable findings: a rapid-cycle evaluation approach to improving implementation. Implementation Science. 2017;12(1): 15 . https://doi.org/10.1186/s13012-017-0550-7

53. Center for Clinical Management Research. Consolidated framework for implementation research 2014 [Available from: http://cfirguide.org/].

54. Greenhalgh T, Robert G, Macfarlane F, Bate $P$, Kyriakidou O. Diffusion of innovations in service organizations: Systematic review and recommendations. The Milbank Quarterly. 2004;82(4):581-629. https://doi.org/10.1111/j.0887-

378X.2004.00325.X

55. Greenhalgh T, Robert G, Macfarlane F, Bate P, Kyriakidou O, Peacock R. Storylines of research in diffusion of innovation: A metanarrative approach to systematic review. Social Science \& Medicine. 2005;61(2):41730.

https://doi.org/10.1016/j.socscimed.2004.12 .001

56. Helfrich CD, Damschroder LJ, Hagedorn HJ, Daggett GS, Sahay A, Ritchie M, et al. A critical synthesis of literature on the promoting action on research implementation in health services (PARIHS) framework. Implementation Science. 2010;5(1):82. https://doi.org/10.1186/1748$5908-5-82$ 